\title{
Current-induced Spin Wave Excitations in Asymmetric Nanopillar Junctions
}

\author{
Arie Fiandimas ${ }^{1}$, Kyung Jin Lee ${ }^{2}$, and Kyung-Ho Shin ${ }^{1 *}$ \\ ${ }^{1}$ Center for Spintronics Research, KIST, Seoul 136-791, Korea \\ ${ }^{2}$ Department of Materials Science and Engineering, Korea University, Seoul 136-73l, Korea
}

(Received 28 January 2009, Received in final form 6 April 2009, Accepted 6 April 2009)

\begin{abstract}
This study examined the current-induced spin wave excitation in asymmetric nanopillar junctions with a stack sequence of $20 \mathrm{~nm} P t / 10 \mathrm{~nm} \mathrm{Cu} / 7 \mathrm{~nm} \mathrm{NiFe} / 300 \mathrm{~nm} \mathrm{Cu}$, and a circular lateral dimension of $240 \mathrm{~nm}$. An analysis of the magnetic and magnetotransport characteristics of the junction showed a possible spin transfer effect at this sample dimension when the magnetization was switched from a vortex state to another state. This finding is expected to help improve the understanding of the spin transfer torque phenomenon in nanopillar junctions.
\end{abstract}

Keywords : nanopillar, magnetotransport, spin transfer torque, spin wave exitation

\section{Introduction}

Current-induced magnetization switching has been demonstrated in a variety of material systems over the last few years. These experiments were based on the seminal predictions by Slonczweski [1] and Berger [2] in that the direct transfer of spin-angular-momentum from conduction electrons to $\mathrm{d}$-spins in a nanomagnet can excite spinwaves, i.e. spin transfer torque (STT). Normally, studies on STT concentrated on the spin valve structure consisting of two ferromagnets. The thicker one provides the spin polarized current that will pass through the thinner one leading to magnetic excitation. However, it was proposed that a polarizer (i.e. the pinned layer) is unnecessary for exciting spin-wave instability [3, 4].

Polianski and Brouwer [3] and Stiles et al. [4] reported that an unpolarized current to the plane of a thin ferromagnetic layer in a normal metal (NM)/ferromagnet (FM)/ normal metal (NM) system can excite the spin-wave instability if there is asymmetry in the pillar junction. Experiments based on this prediction have been demonstrated in a pillar junction containing only a single ferromagnetic layer $[5,6]$.

This study examined the current-induced change in magnetic configuration in asymmetric nanopillar junctions containing only a single circular FM layer, NiFe. A circular

*Corresponding author: Tel: +82-2-958-5418

Fax: +82-2-958-6851, e-mail: kshin@kist.re.kr nanodisk was examined with various thicknesses and lateral dimensions to obtain a better understanding of its magnetization states $[7,8]$. The sample size with dimensions as small as possible was made to have two different magnetization states. All measurements were obtained in various magnetic fields with the field either perpendicular or parallel to the plane geometry at $4.2 \mathrm{~K}$. Magnetic configuration switching was observed at the low current regime in both perpendicular and parallel field geometries. This switching phenomenon was manifested by the changes in resistance near the zero current value. However, for some large magnetic fields in perpendicular geometry, there were distinct changes for only one current polarity. Overall, these results are expected become the first step in the future experiments.

\section{Experimental Procedures}

A new nanostencil mask process [9] was used to fabricate asymmetric pillar junctions with a stack sequence of $20 \mathrm{~nm} \mathrm{Pt} / 10 \mathrm{~nm} \mathrm{Cu} / 7 \mathrm{~nm} \mathrm{NiFe/300} \mathrm{nm} \mathrm{Cu}$, and a circular lateral dimension of $240 \mathrm{~nm}$ (Fig. 1a). NiFe (permalloy) was used as a single ferromagnetic layer. Photoresists were patterned by photolithography onto a $\mathrm{SiO}_{2}$ substrate, which was followed by ion milling and deposition of the bottom electrode. The $\mathrm{SiO}_{2}$ passivation layer was deposited in the following process. The recessed part, $10 \mu \mathrm{m} \times 10 \mu \mathrm{m}$ in size, was formed followed by Pt deposition. Nano-junctions, approximately $240 \mathrm{~nm}$ in diameter, 

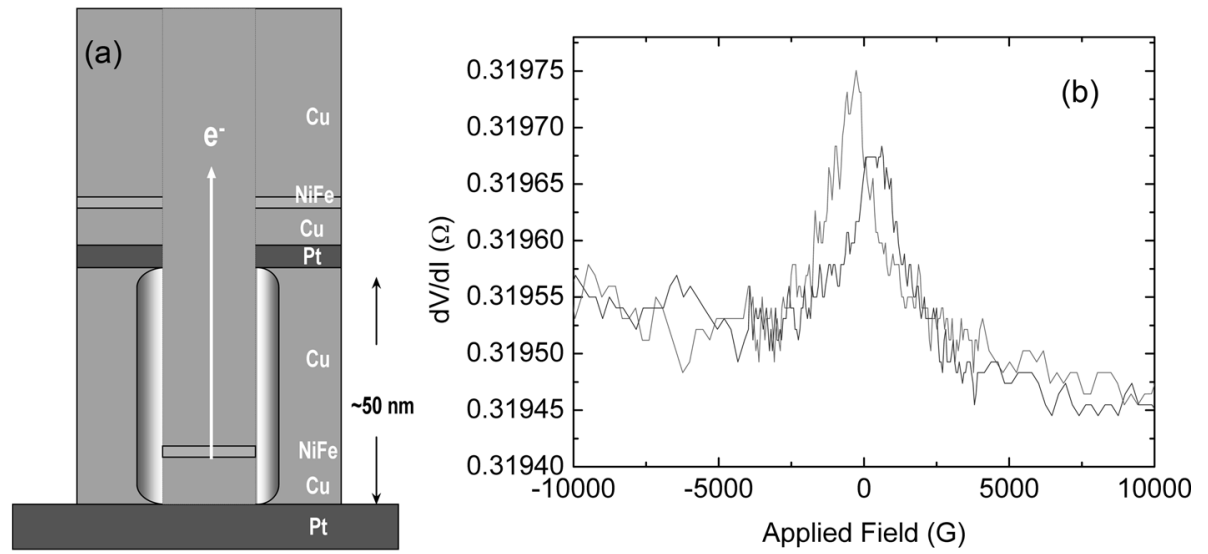

Fig. 1. (a) Schematic diagram of a single and circular NiFe layer pillar junction fabricated using a nanostencil mask process. (b) Typical $\mathrm{dV} / \mathrm{dI}$ vs $\mathrm{H}$ measurements at $1 \mathrm{~mA}$ dc bias and with a perpendicular applied field geometry. The junction resistance changed from a higher value at the near remanence state to a lower value at high field.

were then formed by electron beam lithography and ion milling, in which the positive electron-beam resist PMMA $495 \mathrm{~K} / 950 \mathrm{~K}$ was used as an ion milling mask. A wet etching process was then performed using a HF solution to make a hole under the Pt mask. The stack sequence of $10 \mathrm{~nm} \mathrm{Cu} / 7 \mathrm{~nm} \mathrm{NiFe} / 300 \mathrm{~nm} \mathrm{Cu}$ was then deposited in the hole. Finally, the top electrodes were formed by an ion milling process.

All measurements were carried out at $4.2 \mathrm{~K}$ in a four point-geometry in fields applied perpendicular and parallel to the thin film plane. The differential resistance, $d V / d I$, was measured using a lock-in technique with a $500 \mu \mathrm{A}$ modulation current at $\mathrm{f}=873 \mathrm{~Hz}$ added to a dc bias current. A positive current was defined such that the electrons flow from the bottom electrode of the junction to the top electrode.

\section{Results and Discussion}

Fig. $1 \mathrm{~b}$ shows magnetoresistance measurements of a single layer junction at $1 \mathrm{~mA}$ dc bias. The resistance was high near the remanent state (zero field). The differential resistance decreased with increasing perpendicular field. The resistance became saturated at sufficiently high magnetic fields. At this size of the Permalloy, the magnetizations are in a vortex state [8]. At a high perpendicular field, the magnetic moments were forced to make an angle between the thin film plane and applied field. This may cause a lower resistance at a high perpendicular field [6]. An interesting point in the curve of the differential resistance versus the perpendicular field was that the base line had a nonzero slope (Fig. 1b). This was attributed to the broadening/narrowing of the vortex core depending on the relative direction of the core and field.
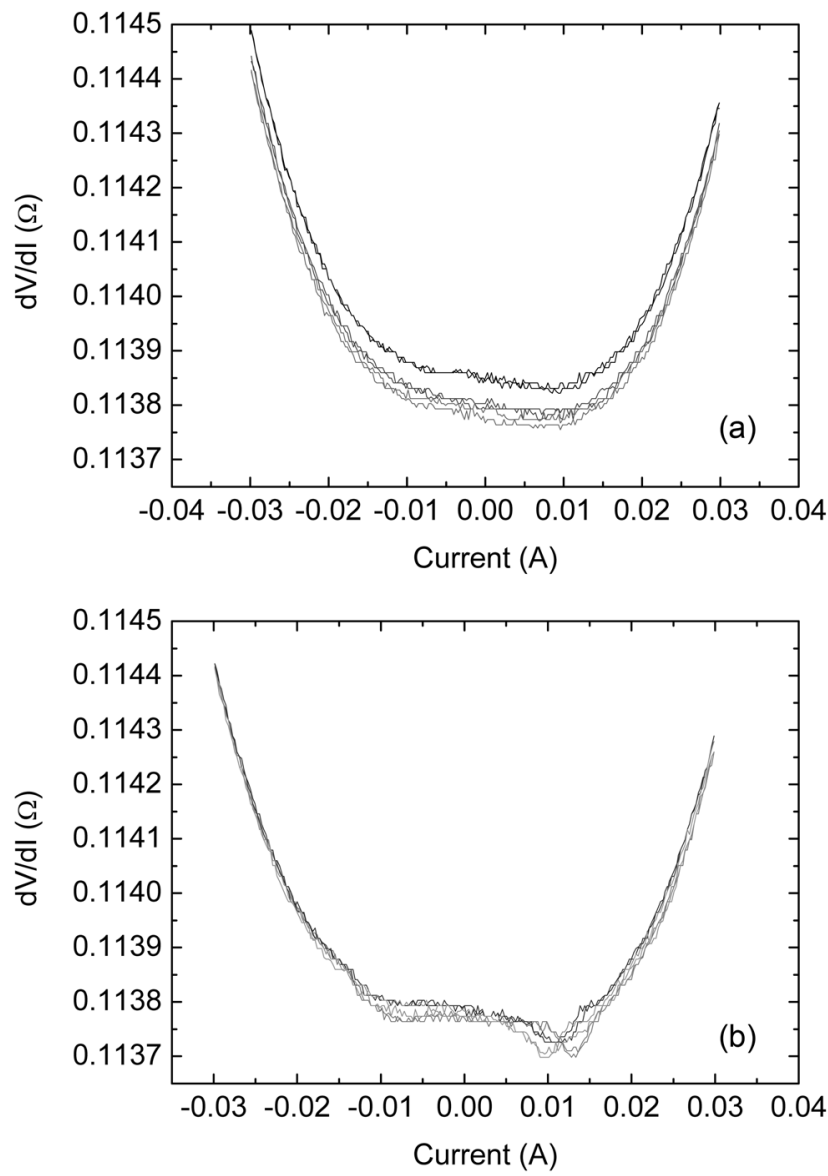

Fig. 2. Current sweeps at a fixed and perpendicular applied field using 3 different samples prepared under the same conditions. (a) At low field, small resistance changes only occur near the transition between positive and negative current polarity. (b) At high field, there are reversible dips in resistance only at positive current polarity. 
Fig. 2 presents a typical $I(V)$ curve with perpendicular magnetic field for an asymmetric nanopillar junction. This plot was obtained in a different sample from that for Fig. 1. Although the same layer structure and patterning methods were applied, there were substantial differences in transport properties according to the sample, possibly due to difference in contact resistance. The $d V / d I$ versus I was plotted for a small field regime, $H=0,2000$, and $4000 \mathrm{G}$ (Fig. 2a). At this regime, a very small resistance change was observed near the zero current. The positive current polarity showed a lower resistance than the negative one. Starting from $+30 \mathrm{~mA}$, the junction remained in a low resistance state until almost $I=+10 \mathrm{~mA}$, where it switched gradually to a higher resistance state. The increase in resistance becomes saturated at almost $I=-2 \mathrm{~mA}$. However, at the higher field regime $(H=8000,9000$, and $10000 \mathrm{G}$ ), reversible small dips were observed at positive current polarity (Fig. 2b), as reported in ref. [5]. These reversible dips tend to move toward the zero current as the magnetic field is increased. This means that the current needed to switch the junction resistance to a low value decreases with increasing applied field. In addition, the critical current to produce this excitation showed a linear dependence on the magnetic field, as reported elsewhere [5].

\section{Conclusion}

This study examined the current-induced spin wave excitation in an asymmetric nanopillar junction with only a single and circular ferromagnetic layer. It was found that at this sample dimension, it was possible to observe a spin transfer effect when the magnetization switched from one vortex state to another, as shown by the resistance changes. This finding is expected to help improve the understanding of the spin transfer effect.

\section{Acknowledgment}

This work was supported by the KIST Institutional program, and by the TND Frontier Project funded by MEST.

\section{References}

[1] J. Slonczweski, J. Magn. Magn. Matter. 159, L1 (1996).

[2] L. Berger, Phys. Rev. B 54, 9353 (1996).

[3] M. L. Polianski and P. W. Brouwer, Phys. Rev. Lett. 92, 026602 (2004).

[4] M. D. Stiles, Jiang Xiao, and A. Zangwill, Phys. Rev. B 69, 054408 (2004).

[5] B. Ozyilmaz, A. D. Kent, J. Z. Sun, M. J. Rooks, and R. H Koch, Phys. Rev. Lett. 93, 176604 (2004).

[6] B. Ozyilmaz and A. D. Kent, Appl. Phys. Lett. 88, 162506 (2006).

[7] M. Heumann, T. Uhlig, and J. Zweck, Phys. Rev. Lett. 94, 077202 (2005).

[8] R. P. Cowburn, D. K. Koltsov, A. O. Adeyeye, M. E. Welland, and D. M. Tricker, Phys. Rev. Lett. 83, 1042 (1999).

[9] J. C. Lee, Master thesis, Inha University, South Korea, 2007. 